\title{
Education as a socio-cultural construction of a person: problems and prospects
}

\author{
Marina Danilova ${ }^{1, *}$, Napoleon Plotnikov ${ }^{1}$ \\ ${ }^{1}$ Kuban State Agrarian University, Krasnodar, Russia
}

\begin{abstract}
Relevance of the topic of work. The problem of worldview factors of social stability has a high degree of relevance in modern society. As part of the work, its aspect is revealed as the possibility of the impact of education on the worldview, depending on the way the educational process is organized. The purpose of the study is the disclosure of the specificity and role of education as a tool for the formation of personal qualities of members of society. Materials and methods. During the preparation of the article, classical scientific methods of analysis, abstraction, and comparison, and synthesis, structural-functional and systemic approaches were applied. The results of the study are related to the analysis of the main constituent elements of the educational process and their actual representation. The study identified key factors in the implementation of the educational function of education related both to the structure of educational programs and to the moment of their demand on the part of students. For discussion, it is proposed to consider the dilemma associated with the choice of priorities of the educational process in the context of a contradiction between the interests of individual students and public interests. Key findings and their significance. In the process of research, the importance of education as a mechanism for shaping the worldview was determined. The social risks associated with this process are identified, and key opportunities for improving the effectiveness of the humanitarian component of the educational process in modern society are identified.
\end{abstract}

\section{Introduction}

The judgment that man is the product of culture is well known. At the same time, a person is never a product of the whole culture for the simple reason that the cognitive abilities of a person do not cover the totality of cultural phenomena. Thus, when we say that a person is a product of culture, we mean the cultural situation in which he is included. And this is of fundamental importance, since within the framework of the same cultural environment there can be radically different selections of its elements that have a formative effect on the worldview of the subject. In other words, different cultural situations determine different forms of worldview, and, therefore, heterogeneity of culture defines a wide range of worldview forms. This is the reason why reality is often far from its generalized picture. And this is the reason why, despite all the achievements of social knowledge, the moment of

\footnotetext{
*Corresponding author: madary@mail.ru
} 
conflict between a person and society remains, manifested in such outwardly destructive forms as civil disobedience and crime, as well as in destructiveness that a person turns to himself. In this case, we focus on the fact that this contradiction may have a local character, not structurally, but culturally.

At the early stages of the development of society, when autonomy of the individual was not yet developed, constitutive social principles were of predominant importance, and a person acted as an object and "material" of social processes. However, as social development proceeds, a steady change in the situation occurs in which the "compliance" of a person with respect to the interests of society is significantly reduced.

Society seeks to take control of the areas of social reality on which it is most dependent. However, there is no doubt that the nature of the social worldview of members of society is one of the key factors in the existence of the social system. Therefore, in addition to the natural, self-regulating sphere of culture, there are also centralized mechanisms of influence on the worldview of members of society, designed to inculcate a certain set of norms, values, behaviors, methods of self-identification, etc. One of such mechanisms is education, whose high role in socialization is not is subject to doubt. In this article, we focus on revealing the function of education related to the formation of socially significant qualities of a person through the creation of a specific cultural situation in the learning process that affects the worldview of children and young people. One of the key ideas developed in the framework of the work is the idea of constructing a person on the basis of his ideal model, which forms the basis of the formed educational process.

\section{Purpose of the study}

In this work, we are focused on revealing the specifics and role of education as a tool for the formation of personal qualities of members of society. To this end, the general functional certainty of education is investigated, the humanitarian and social aspects of educational activity are detailed, and the mechanisms of socialization and social control implemented directly by teachers are analyzed. We also study the indirect educational impact, carried out by introducing students to various cultural achievements.

\section{Materials and methods}

In the process of research, research is supported by T.T Cherkashina, Yu.V. Chernova, N.S. Novikova [1], V.V. Morozova [2], E.N. Shapinskaya, A.A. Lisenkova [3], A.D. Gurarii [4]. Also, in the course of the work, the results of previous author's studies were used $[5,6]$. Of great importance in the preparation of the study were the works devoted to the humanitarian aspect of educational activity in the modern world, including the studies of such authors as T.D. Namwambah [7] I. Liashchynskaya, V. Jakubovská [8], S. K. Berdibayeva, S. M. Maigeldiyeva, S. G. Tazhbayeva, M. A. Abirova, S. K. Atakhanova [9], A. Csikosova, K. Teplicka, A. Senova [10], H. A. Ead [11], I. Gürşimşek, M. Göregenli [12], A. Kornienko [13], V. J. Llorent [14], O. Loyko, S. Dryga, J. Park, M. Palianov [15], F. Reichert, J. TorneyPurta [16], M. Voinea [17], A. Vorontsov, Vorontsova E. [18].

In the course of preparing the article, classical scientific methods of analysis, abstraction, comparison, synthesis were applied. Also, the need to reflect the functional aspect of educational activity determined the appeal to the structural-functional approach. Also, at some stages of the study, a systematic approach was applied. 


\section{The results of the study}

The basic model for the consideration of education suggests that it is two-component in its functional orientation. On the one hand, education is focused on learning, which is a combination of transferred knowledge and generated skills. On the other hand, education is an educational tool, which involves working on the nature of the social worldview and includes the formation of value attitudes, inculcation of behaviors, methods of social selfidentification, criteria for assessing environmental reality, etc.

The balance between educational and training activities in the educational process is one of the important problems in the field of pedagogy. At the same time, in essence, we are talking about two areas of impact on the subjects of educational activity - aimed at their development and aimed at their formation (limitation). The efforts spent on educating young people are in the public interest. It is about the formation of worldview qualities that are compatible with the existing social order. To a certain extent, one can also judge that upbringing contributes to a more effective adaptation of young people to the surrounding reality, into which they, accordingly, are more organically integrated. However, here the priority is precisely social interests related to the inclusion of the subject in a streamlined system of interaction. In turn, training contributes to personal development, which is in the student's area of interest, since it is about expanding his knowledge of the world around him, as well as expanding the tools available to him skills. In relation to the question raised in this discussion, the question of the fact that various modes of the educational process may take place is of interest. The first option is associated with the direct transfer of knowledge, which implies a moment of high speed of information transmission, while the second option is expressed in creating the conditions for students to independently discover certain principles and patterns. In the first case, we observe significantly lower time costs, however, it is associated with a significant risk of stereotyping of education. At the same time, the second version of the teaching activity, although it does not set the conditions for the rapid transfer of knowledge, however, it contributes to the development of cognitive abilities of students. The presented reasoning serves as an illustration of the moment of multivariant organization of the educational process.

It should immediately be noted that the above classification of the functions of education is illustrative and does not reflect reality in all its complexity. Education and upbringing are rather the various priorities of educational activity that determine differences in its format and content of the taught material. The separation between them is not absolute - which we will dwell on later in the framework of the analysis of the possible prospects for the development of the educational sphere. At the same time, the above-described discrepancy in the vectors of the organization of the educational process takes place, and, moreover, within the framework of constructing educational programs, they proceed from it, making a choice of priorities for educational activities. And, accordingly, we can observe various examples of the organization of educational institutions in which educational activities were paid more or less attention. In this case, the question of whether it is possible to build a "pure" educational process, expressed at the level of a specific social function, is of interest. In our opinion, the ratio of training and education is, first of all, a feature of the humanitarian program, or a mixed form of training. Technical and natural sciences do not contain an educational context, and therefore, at their level, training in its pure form can be implemented without the addition of educational elements. In turn, humanitarian education already allows the question to be asked about the relationship between an action that shapes a person and an action that fills the mind in the educational process. Accordingly, when we raise the question of the correlation of educational and educational aspects, we either talk about the correlation of humanitarian and natural science (or technical) knowledge, or we raise the question of the independent activity of a teacher as a carrier of educational function. 
Before proceeding to the consideration of the theoretical aspects of the social construction of a person through the education system, attention should be paid to the fact that education can be focused on the formation of a stable set of external rituals, or focus on changing personal characteristics of students. Parenting is an extremely complex phenomenon, various aspects of which can be represented to varying degrees. This is one of the reasons why the focus of this work is on the sociocultural construction of a person in the process of educational activity. We intentionally narrow down the study area of the educational environment in order to avoid possible discrepancies. In this regard, such moments as drill, instilling discipline, the implementation of relationships in a vertical hierarchical model - all this falls outside the scope of consideration, referring more to the topic of the status-role aspect of relations in an educational institution than to the sociocultural impact on the worldview.

Let us return to the question of the structure of educational activities related to the formation of certain aspects of the worldview through familiarization with specific cultural achievements. It should be noted here that there are three dimensions of educational activity that contribute to the inclusion of the student in the cultural environment, and, through this, the formation of his worldview. Among them stand out:

- Familiarization with the achievements of artistic culture and various fields of art (both classical and contemporary). This includes not only acquaintance with painting, music and architecture, but also the expansion of literary horizons. Reading the world classics (provided that students are ready for such an experience, but do not acquire it in a forced form) can have a powerful impact on the nature of the worldview, which is associated with the moment of indirect experience of the experience of key characters, as well as the familiarization with their reflective activities, valuable installations, etc. It should be noted that at the present stage of this kind of impact on the worldview can be carried out, including within the framework of such a direction of cultural creativity as cinema. Moreover, it is noteworthy that, despite the centuries-old history of cinema, most educational programs do not pay attention to this layer of culture;

- The study of social sciences (this includes history, law, sociology, economics, social psychology, political science, social philosophy, and other sciences). This direction is of great importance in the development of the social worldview due to the fact that within its framework extensive knowledge is given about various areas of public life, moreover in an orderly and systematized way. And, simultaneously with the moment of social cognition, in this case, an axiological approach is implemented, associated with the formation of attitudes to certain events and phenomena of public life. It is no coincidence, for example, that the study of history is one of the powerful mechanisms for raising the level of civic consciousness. Here, there is an actualization of certain aspects of the subject's selfidentification in the process of his educational process. Describing at a general level the role of social sciences in the education of young people, it should be noted that their study is an indirect form of cognition of social reality. And therefore, the humanitarian aspect of education is fundamental in shaping the social worldview of members of society;

- The formation of the ability to independently adequately assess social reality and, in particular, cultural phenomena that a student is faced with for one reason or another. If the two aspects indicated above characterize the student's familiarization with certain elements of culture (that is, the creation of the same cultural situation in which the formation of certain worldviews is logical), as well as the formation of a certain paradigm of perception of social reality, then in this case we mean dynamic aspect of the social worldview associated with the ability to make independent judgments about the surrounding reality. As a matter of fact, the last of the considered options for education consists in the formation of a developed mind, which makes it possible to critically conceptualize certain cultural phenomena that a person 
encounters in the course of his life, to determine their value component, consequences for society, etc.

We deliberately included in the thematic consideration of the possibilities of education for upbringing through the creation of a cultural situation, such an aspect as familiarization with the sciences and scientific methodology. Here I would like to focus on the fact that science is also a component of culture, and, accordingly, the measure of involvement in the humanities is one of the factors of the cultural plan that determine the worldview of a person. For this reason, humanitarian knowledge is one of the most important components of education, focused not only on the creation of a set of knowledge and skills that have a practical orientation, but also on the formation of a set of qualities of students necessary for their more successful integration into the public environment.

At first glance, this kind of model sets an effective scheme of educational activity related to the inclusion of a humanitarian program in the educational process, regardless of what specialty education takes place (if we are talking about studying at universities or secondary schools with a bias in a certain disciplinary direction). Humanitarian knowledge taught in schools and universities becomes that universal cultural context in which the moment of formation of the worldview of members of society is realized. As a result, the education system influences the processes of formation of the social picture of the world, the formation (or correction) of the value structure of the personality, the adoption of behavioral models and methods of implementing identification and self-identification processes. However, such an effective impact of education on the worldview is possible only if certain conditions are met, among which one can distinguish:

- The relevance of the cultural context of human development, defined by the educational environment, in comparison with other cultural situations in which the person is involved;

- The demand for education (which includes both recognition of the importance of the educational sphere in principle, and the demand for taught humanitarian knowledge).

Consider in order the identified aspects of the success of the educational impact on the personal development of members of society. Regarding the relevance of the cultural context, we are talking about the relationship between the educational environment and other channels of cultural and informational interaction, both at the level of priority of perceived meanings and ideas for the worldview, and in terms of the degree of intensity of influence on the worldview of a person. Education used to be one of the main channels for acquiring knowledge; in a number of aspects it was uncontested. This determined the moment of exclusivity of the educational process and the desire for the effectiveness of capturing the knowledge gained in its course. The educational environment turned into a specific cultural layer, in the framework of which its priorities and values related to the academic sphere were present. The democratization of education, as well as the formation of an increased number of study places, determined the accessibility of education; At the same time, over the past decades, the process of information exchange growth has gradually been unfolding, which is associated with the intensification of cultural and information communication in the framework of broadcasting and, in the future, intensive Internet communication. Nowadays, the exclusivity of knowledge provided within the framework of the information environment is gradually being lost. Education is no longer a way to overcome information hunger; in the conditions of modern information technologies it was replaced by the satiation with a large amount of information. Moreover, official educational institutions, and, in particular, lectures given by specialists are of interest to students on the following grounds:

- The ability to obtain expert, in-depth knowledge in a particular subject;

- Viewing the educational course as a performance (which implies the serious importance of the image aspect of the teacher's activities, his charisma and the way the material is presented);

- The need to implement the formal aspect of educational activity. 
Currently, the density of cultural and informational interaction is extremely high, in fact - it has peak indicators in comparison with all known eras. It is a question of both information exchange processes within the same cultural environment and intensive intercultural communication [8]. Moreover, most of a person's information needs can be satisfied within a few seconds within the network sphere, which brings social knowledge mediated by network technologies to an extremely high level [6]. We do not take the deepest and most complex scientific and professional questions, although a significant amount of network materials can also be found on them (for example, through banks of dissertations, libraries of educational materials, etc.). People interact with each other at the everyday and informal levels, consume a huge amount of entertainment and educational information products, and all this leaves a serious imprint on their worldview. In today's world, the educational environment is only one of a large number of sources of worldview formation. And if we raise the question of the importance of education as an instrument for the formation of a certain set of human qualities, we have to admit that currently there is competition between education and a wide range of information areas. In other words, the paradigm of considering education as a mechanism for the socio-cultural construction of a person requires substantial improvement in connection with the current situation in the cultural and information environment.

The second of the conditions for the effectiveness of the formative activity of education is also in doubt due to modern transformational processes in society. Education must be in demand so that it can influence the worldview of members of society. And since we are talking about the relevance of humanitarian education, we should pay attention to the serious trend of the deactivation of humanitarian knowledge as part of the educational process. Let us turn to this question in more detail.

We have already said above that it is humanitarian education that can act as that universal (due to the centralized nature of the educational system) cultural situation that contributes to the consolidation of members of society and their more effective inclusion in the social environment. However, additional education is, at the same time, an additional burden, or an increase in the educational process. And the more complex and dynamic the fields of activity studied in higher education are, the more acute is the question of how much they need subjects that are not directly related to their professional profile. We have already noted the fact that education is in the sphere of interests of students, whereas education reflects the interests of a higher order. The problem is that students' understanding of this conflict entails, as one of the results, a decrease in motivation in those subjects that belong to the non-core field. This aspect as a whole can be designated as a tendency to deactivate humanitarian education.

The second trend, which generally reduces the ability to implement a cultural impact on the worldview of students, is that in addition to developed universities, numerous training courses are being formed, the main advantage of which is integrity and conciseness associated with an orientation toward transferring exclusively specialized knowledge. Taking such courses significantly saves students time, making the educational process more effective in terms of the dynamics of professional development. For some modern professions this is necessary due to the extremely high dynamics of the latter, since the knowledge acquired several years earlier has time to become obsolete and, accordingly, require revision. The problem is that the increasing complexity of existing specialties, as well as the tendency for narrowly specialized courses to be formed, contributes to the displacement of humanitarian subjects from, at least, the attention of students, and, as a maximum, from the institutional system of vocational education [7]. 


\section{Discussions}

The dilemma of choosing an educational strategy is connected with the discrepancy between the field of interests of students, among which the profile professional education is of dominant importance, and the sphere of social interests. Moreover, in some cases, the formation of a comprehensive humanitarian course within the framework of the educational program makes the latter cumbersome and, accordingly, extended in time. This leads to the fact that education is losing its effectiveness in the aspect that is associated with the learning process. In turn, the tendency to minimize the socio-humanitarian aspect of educational activity entails negative consequences for society. This is a fundamental contradiction that defines diametrically opposite positions in the discussion of the future educational sphere and the priorities of its development and transformation. Accordingly, the question remains on the agenda of which aspect of educational activity is a priority and, in turn, how to organize the educational process in such a way as to increase its effectiveness and minimize losses in functionality.

\section{Conclusions}

The above trends allow us to judge that the degree of influence of education on the social worldview and personal qualities of members of society in recent decades has been significantly reduced. Under these conditions, the question of how the realization of the education function is possible, related to the integration of society into the social environment and, in particular, the formation of a number of qualities necessary for the formation of an adequate vector of social development, is becoming relevant. In fact, we are talking about how possible the implementation of the educational function related to the socio-cultural construction of man is currently possible. To answer this question, it is necessary to consider more deeply the correlation of education and training, as well as the ratio of active activity and passive perception in the educational process.

The moment has already been indicated above, according to which there is no direct contradiction between the educational process and the learning process, in other words, situations are possible when they are combined. This is especially true in view of the fact that the knowledge of the world (including the sociocultural sphere) contains a moment of individual activity related to the interpretation and reflective reflection of the gained experience and knowledge. And in this case, cognitive abilities (which include, inter alia, acquired mental skills and methods of cognition) determine the nature of the relationship to a particular acquired knowledge, as well as the results of familiarizing with it. There is a range from misunderstanding to in-depth understanding on the one hand and from noncritical acceptance to consciously questioning, on the other. The ability of cognition and the existing worldview is the filter that determines the nature of the knowledge and ideas accepted in the future.

Here it is worth focusing on another aspect. The concept, which assumes the planned formative impact on the members of society through the educational sphere, considers students, first of all, as formed material, in other words, the object of influence. At the same time, in a modern form, it is advisable to consider education rather as a dialogue in which both sides manifest their subjectivity. With this approach, we can talk not so much about instilling certain qualities, but about creating the conditions for their formation. It should be noted separately that the attitude towards students as a material, an object of influence, denies their individuality and freedom, which in itself is problematic from the point of view of the ethical aspect of the action taken.

Regarding the reduction in the relative amount of culturally significant information transmitted during the educational process, as well as the sharp intensification of third-party 
information processes, we note that, in our opinion, it is extremely difficult for education to compete with other spontaneously emerging areas of culture, especially considering that the struggle for attention is one of their organizing principles. In a number of cases, education cannot provide extensive familiarization with the elements of culture that carry constructive models of action, ideas, and values. At the same time, it can provide a methodology for understanding the surrounding cultural environment, which makes a person substantially protected from its elemental influences and allows him to independently come to an understanding of a number of socially significant attitudes. In other words, one of the priorities of modern humanitarian education is the development of the subject of social cognition, and not the filling of its worldview. This can contribute to raising awareness of the social activity of members of society, and their transition to constructive models not because of the manipulations carried out against them, but within the framework of a personal, informed choice.

The attractiveness of this path is largely due to the fact that it allows you to form an active, interested attitude of students to the surrounding life, which to a large extent helps to overcome existing illusions and myths, with a further understanding of the semantic connections and patterns that lie behind them. In such a situation, humanitarian knowledge moves from the status imposed on students into the field of interests, which sets broad prospects.

In this case, the question remains acute at what stage of educational activity of this kind an approach can be most effective. There are several possible ways:

- The introduction of advanced courses in the methodology of humanitarian knowledge in school practice;

- The formation of materials focused on the popularization of humanitarian knowledge (including those distributed in the public domain);

- The inclusion of a course focused on deepening the skills of humanitarian knowledge in existing university courses.

The question of choosing a way to implement this program is worthy of a separate study. At the same time, there is no doubt that the humanitarian function of education must adapt to modern sociocultural transformations, otherwise the formative effect of education on members of society can practically come to naught.

\section{References}

1. T.T. Cherkashina, Yu.V. Chernova, N.S. Novikova, Bulletin of the Orenburg State University 3(221), 79-86 (2019) DOI: 10.25198 / 1814-6457-221-79

2. V.V. Morozov, Historical and socio-educational thought 3 (2015) DOI: 10.17748 / 2075 9908.2015.7.3.233-238

3. E.N. Shapinskaya, A.A. Lisenkova, Cultural code 1, $42-54$ (2020) DOI: 10.36945 / 2658-3852-2020-1-42-54

4. A.D. Gurariy, Sociodynamics 7, 10-20 (2019) DOI: 10.25136/ 2409-7144.2019.7.29179

5. M.I. Danilova, N.V. Isakova, G.M. Nikitin, E.V. Yakovleva, European Proceedings of Social and Behavioural Sciences 39, 337-345 (2019) DOI: https://doi.org/10.15405/epsbs.2019.03.02.39

6. M. Danilova, G. Blokhovtsova, V. Plotnikov, N. Plotnikov, The European Proceedings of Social \& Behavioural Sciences EpSBS LXXVI -SCTCMG(88), 652-660 (2019) DOI: https://doi.org/10.15405/epsbs.2019.12.04.88

7. T.D. Namwambah, International Journal of Humanities Social Sciences and Education (IJHSSE) 7(3), 90-97 (2020) DOI http: // dx. doi.org/10.20431/2349-0381.0703011 
8. I. Liashchynskaya, V. Jakubovská, Konštantínove listy 10(2), 191-203 (2017) DOI https://doi.org/10.17846/cl.2017.10.2.191- 203

9. S.K. Berdibayeva, S.M. Maigeldiyeva, S.G. Tazhbayeva, M.A. Abirova, S.K. Atakhanova, Procedia - Social and Behavioral Science 11621, 2941-2945 (2014) DOI: 10.1016/j.sbspro.2014.01.684

10. A. Csikosova, K. Teplicka, A. Senova, Procedia - Social and Behavioral Sciences 462012, 2978-2982 (2012) DOI:10.1016/j.sbspro.2012.05.600

11. H.A. Ead, Research in Globalization 1(100003) (2019) DOI: http://dx.doi.org/10.1016/j.resglo.2019.100003

12. I. Gürşimşek, M. Göregenli, Procedia - Social and Behavioral Sciences 1(12009), 975979 (2009) DOI:10.1016/j.sbspro.2009.01.173

13. A. Kornienko, Procedia - Social and Behavioral Sciences 20617, 359-364 (2015) DOI: 10.1016/j.sbspro.2015.10.065

14. V.J. Llorent, Procedia - Social and Behavioral Sciences 462012, 3619-3623 (2012) DOI: 10.1016/j.sbspro.2012.06.116

15. O. Loyko, S. Dryga, J. Park, M. Palianov, Procedia - Social and Behavioral Sciences 20617, 464-468 (2015) doi: 10.1016/j.sbspro.2015.10.084

16. F. Reichert, J. Torney-Purta, Teaching and Teacher Education 77, 112-125 (2019) DOI: https://doi.org/10.1016/j.tate.2018.09.005

17. M. Voinea, Procedia - Social and Behavioral Sciences 332012, 288-292 (2012) DOI:10.1016/j.sbspro.2012.01.129

18. A. Vorontsov, E. Vorontsova, Procedia - Social and Behavioral Sciences 2145, 1147 1155 (2015) DOI: 10.1016/j.sbspro.2015.11.731 